Check for updates

Cite this: RSC Adv., 2017, 7, 50781

Received 4th September 2017

Accepted 23rd October 2017

DOI: 10.1039/c7ra09813h

rsc.li/rsc-advances

\section{Controlled growth of aligned GaN nanostructures: from nanowires and needles to micro-rods on a single substrate $\uparrow$}

\author{
Danna Zhao, (D) Hui Huang, * Rui Lv, Shunji Chen, Qiyilan Guang, Yang Zong, Zhe Liu \\ and Xiqing Li
}

Simultaneous growth of different kinds of aligned GaN nanostructures (i.e., nanowires, needles, pyramids and micro-rods) on a single substrate was firstly realized at a low temperature of $790{ }^{\circ} \mathrm{C}$ by naturally changing the III/V ratio across the substrate via a coaxial pipeline configuration. The effects of substrate distance and growth pressure on nanostructure growth were investigated. The morphology variation from nanowires to micros-rods would be explained in terms of Ga species changing from the Ga element to GaN molecule in a hot-wall reactor. This work is helpful for on chip integration of different kinds of nanodevices on unusual substrates of low melting temperature.

\section{Introduction}

Semiconductor nanostructures, such as nanowires, ${ }^{\mathbf{1 - 3 , 1 2 - 1 5 , 2 4 - 2 9}}$ needles ${ }^{4-6}$ pyramids, ${ }^{7,8,16,38}$ rod/pillars, ${ }^{\mathbf{8 , 9 , 1 6 - 2 3}}$ and trumpets, ${ }^{\mathbf{1 0 , 1 1}}$ have wide applications in novel electronic devices. In comparison with the nanowire structure, ${ }^{\mathbf{1 - 3 , 1 2 - 1 5 , 2 7 , 2 9}}$ vertically aligned needle, rod and pyramid structures, which have a nanoscale tip and robust microscale-trunk, can bring advantages in single nano-structure devices ${ }^{4-6}$ and optical coupling. ${ }^{10,11}$

Currently, metal-organic chemical vapor deposition (MOCVD) is one of the most popular techniques for commercialized GaN epitaxy. For MOCVD growth of GaN nanostructures, there has two main methods, i.e., vaporliquid(solid)-solid growth with metal catalyst (VLS) 1,2,12,13,24-27,29 and catalyst-free anisotropic-growth on patterned substrate (i.e., selective area growth (SAG))..$^{7-9,16-23}$ However, for the nanowires with high aspect ratio (length/diameter $>35$ ), it is difficult to be grown via SAG method $^{7-9,16-18,22,23,39}$ without the use of high silane flux ${ }^{18,20}$ or complex pulsed-growth mode. ${ }^{24}$ While, for the micro-rods of low aspect ratio (i.e., microscale-trunk rods), there is few report that they can be grown via VLS method., ${ }^{1,2,12,13,24-27,29}$ In other word, neither VLS nor SAG can be satisfactorily used for growth of both nanowires and micro-rods.

Department of Electronic Science and Technology, Faculty of Electronic Information and Electrical Engineering, Dalian University of Technology, Dalian 116024, China. E-mail: huihuang@dlut.edu.cn

$\dagger$ Electronic supplementary information (ESI) available: S1-S2 Transmission electron microscopy (TEM) analyses of nanowire and pyramid from sample A. S3-S4 The TEM analyses of the triangular needle and hexagonal rod from sample B1. S5 The TEM analyses of the hexagonal rod from sample B2. S6 The X-ray diffraction spectra of sample B1, sample B2 and substrate. S7 The photoluminescence (PL) spectra of nanowires, needles and micro-rods. See DOI: 10.1039/c7ra09813h
Moreover, for different nanostructures, there exists large difference in growth conditions (especially in the growth temperature). Kuykendall et $a .^{2}{ }^{2}$ achieved nanowire arrays via VLS at $780{ }^{\circ} \mathrm{C}$. Tian et al. ${ }^{36}$ prepared micro-pyramids via SAG at a high temperature about $1080{ }^{\circ} \mathrm{C}$. Bae et al. ${ }^{37}$ investigated the morphologies of micro-pyramids, and they found that micropyramids with smooth sidewalls can only be grown at the temperature higher than $900{ }^{\circ} \mathrm{C}$. Rozhavskaya et al. ${ }^{3}$ reported the growth of micro-rod arrays under $1040{ }^{\circ} \mathrm{C}$. Thus, growth temperature of these micro-structures prepared via SAG (950$\left.1175{ }^{\circ} \mathrm{C}\right)^{7-9,16-18,22,23}$ is much higher than that of nanowires prepared via VLS $\left(760-850{ }^{\circ} \mathrm{C}\right) .^{\mathbf{1 , 2 , 1 2 , 1 3 , 2 4 - 2 7 , 2 9}}$ Thus, it is still a challenge to simultaneous growth of all these nanostructures (including nanowires and micro-rods) under a favorable condition.

In this paper, aligned GaN nanowires, needles, pyramids and micro-rods can be grown simultaneously on a single substrate at a constant temperature of $790{ }^{\circ} \mathrm{C}$, by naturally changing the V/III ratio across the substrate. The growth mechanism, which induces the transformation between these nanostructures, was also discussed.

\section{Experiment}

Growth of the GaN nanostructures was performed by using a homemade hot-wall MOCVD system, in which the growth parameters can be flexibly tuned. As shown in Fig. 1(a), the substrate distance (defined as the distance between the substrate and the outlet of quartz coaxial pipeline) can be changed. The coaxial pipeline contains an inner-tube $(4 \mathrm{~mm}$ i.d., $5 \mathrm{~mm}$ o.d.) and an outer-tube (7 mm i.d., $8 \mathrm{~mm}$ o.d.), which is used for delivering TMGa and $\mathrm{NH}_{3}$ to the substrate, respectively. This coaxial pipeline configuration results in a gradual 

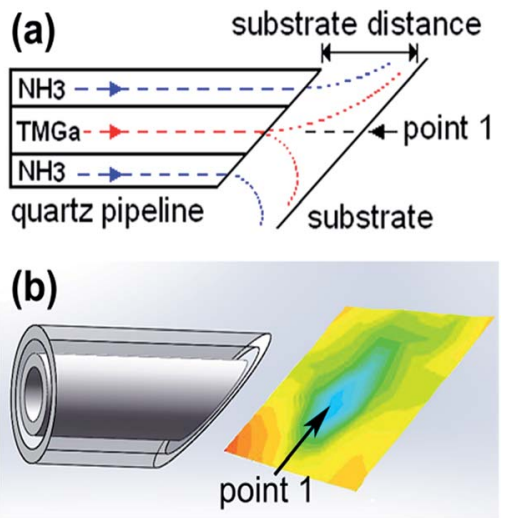

Fig. 1 (a) Schematic diagram and (b) gas distributions of the coaxial pipeline.

Table 1 Growth parameters of GaN samples

\begin{tabular}{lllll}
\hline Sample & $\begin{array}{l}\text { TMGa } \\
{[\mu \mathrm{mol}} \\
\left.\mathrm{min}^{-1}\right]\end{array}$ & $\begin{array}{l}\mathrm{NH}_{3} \\
{\left[\mathrm{mmol}^{-1}\right.}\end{array}$ & $\begin{array}{l}\text { Pressure } \\
{[\mathrm{Torr}]}\end{array}$ & $\begin{array}{l}\text { Substrate } \\
\text { distance } \\
{[\mathrm{mm}]}\end{array}$ \\
\hline A & & & 100 & 8 \\
B1 & 9 & 1.8 & 100 & 18 \\
B2 & & & 200 & 18 \\
\hline
\end{tabular}

decrease of the III/V ratio from the center (point $1 \#$ ) to the edge of the substrate, while the other growth parameters can remain the same across the substrate. Thus, the effect of III/V ratio on nanostructure growth can be found out on a single substrate, by carrying out only a single growth, which can eliminate the run to run variation.

As listed in Table 1, three samples were grown at an optimized temperature of $790{ }^{\circ} \mathrm{C}$ for $15 \mathrm{~min}$. The flow rate of $\mathrm{N}_{2} /$ $(5 \%) \mathrm{H}_{2}$ gas used for carrying $\mathrm{TMGa}$ and $\mathrm{NH}_{3}$ precursors was 350 sccm and $150 \mathrm{sccm}$, respectively. (0001) sapphire wafer coated with a $3 \mu \mathrm{m}$ (0001) GaN-layer was employed as substrate. ( $2 \mathrm{~nm}$ ) $\mathrm{Ni} /(2 \mathrm{~nm}) \mathrm{Au}$ catalyst layers were successively deposited on the substrate by magnetron sputtering.

\section{Results}

\section{Sample A}

Fig. 2(a) shows the optical photograph of sample A, which was grown with a substrate distance of $8 \mathrm{~mm}$. With such a short distance, the mixture between $\mathrm{TMGa}$ and $\mathrm{NH}_{3}$ precursors was far from homogeneous when they arriving the substrate, so the III/V ratio can change greatly across the substrate. As shown in Fig. 2(a), with moving from the center (point 1\#) to the edge (point 5\#) of the growth region (indicated by the red circle) on the substrate, the surface color changes from black (1\#), yellow $(2 \#, 3 \#)$, to light yellow ( $4 \#, 5 \#)$. The black-disk (around point $1 \#)$ is the region, which directly faces the inner-tube of the coaxial pipeline (Fig. 1).

Fig. 2(b)-(f) show the scanning electron microscopy (SEM) images of the five points $(1 \#-5 \#)$ of sample A, respectively. At

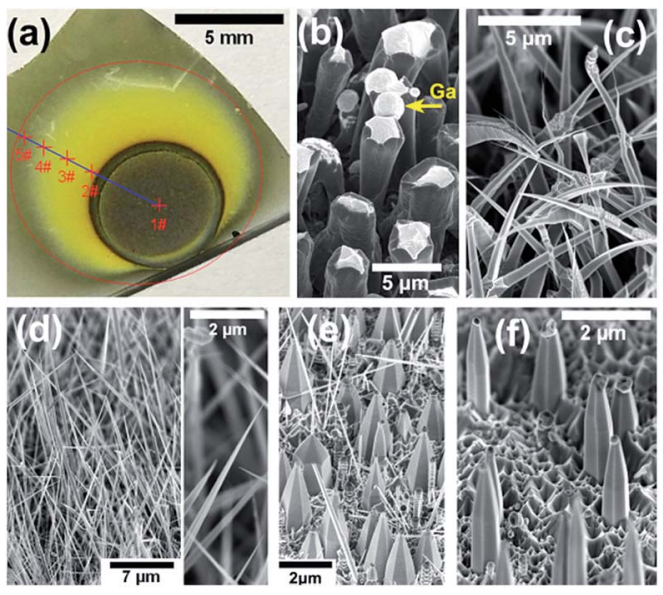

Fig. 2 (a) The optical photograph and the SEM images of point (b) 1\#, (c) 2\#, (d) 3\#, (e) 4\#, and (f) $5 \#$ of sample A.

point 1\# (Fig. 2(b)), distorted large rods were observed with white cap (indicated by an arrow), which could be induced by excess supply of Ga species, because of extremely high III/V ratio at the region of the black-disk. At point $2 \#$ (Fig. 2(c)), curve needles (base diameter of $\sim 700 \mathrm{~nm}$ and length of $\sim 20 \mu \mathrm{m}$ ) were observed without excess Ga at the tip. At point 3\# (Fig. 2(d)), oriented triangular nanowires with diameter of $\sim 200 \mathrm{~nm}$ and length of $\sim 20 \mu \mathrm{m}$ were observed. At point $4 \#$ (Fig. 2(e)), vertical hexagonal pyramids with base diameter of $\sim 1.5 \mu \mathrm{m}$ and length of $\sim 4 \mu \mathrm{m}$ were observed. At point 5\# (Fig. 2(f)), vertical hexagonal micro-rods with trunk diameter of $\sim 0.7 \mu \mathrm{m}$ and length of $\sim 3 \mu \mathrm{m}$ were observed. At the points outside the growth region, nanostructures are very sparse due to lack of Ga species (i.e., extremely low III/V ratio). Thus, by simply decreasing the III/V ratio across the substrate, the nanostructures of needles (2\#), nanowires (3\#), pyramids (4\#), or micro-rods (5\#) can be obtained respectively. As shown in the S1-S2 of the ESI, $\dagger$ the

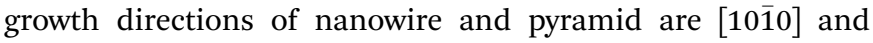
[0001], respectively.

\section{Sample B1}

Fig. 3(a) shows the optical photograph of sample B1, which was grown with an increased substrate distance of $18 \mathrm{~mm}$. The increased distance would enhance the mixture of TMGa and $\mathrm{NH}_{3}$ precursors before they arrive the substrate, so more
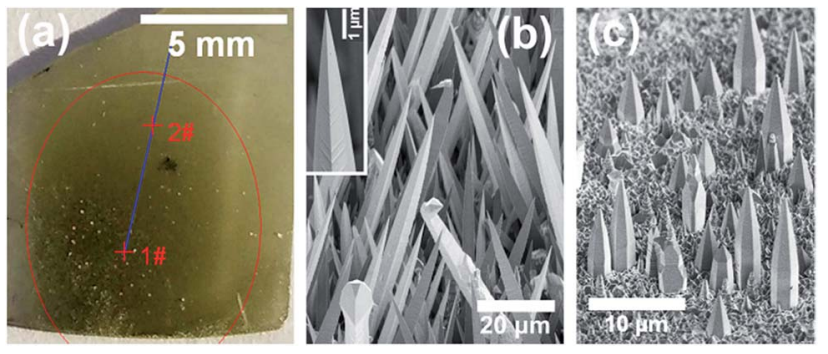

Fig. 3 (a) The optical photograph and the SEM images at point (b) 1\#, (c) 2\# of sample B1. 


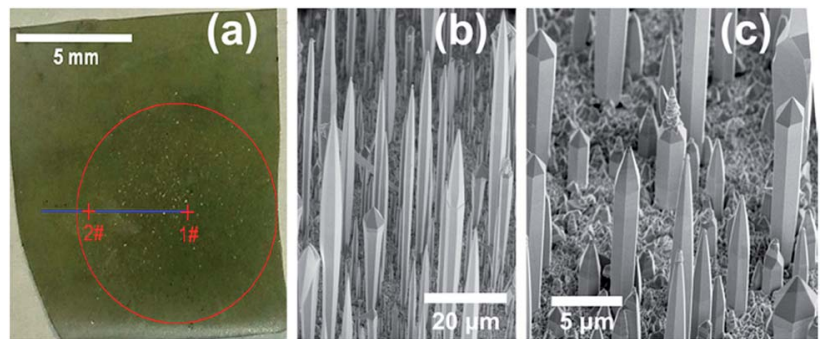

Fig. 4 (a) The optical photograph and the SEM images at point (b) 1\#, (c) 2\# of sample B2.

uniform distribution of the nanostructures across the substrate can be obtained. As shown in Fig. 3(a), only yellow-green color was observed, and the color depth decreases slightly from the center (point 1\#) to the edge (point 2\#) of the growth region.

Fig. 3(b) and (c) shows the SEM images of sample B1. At point 1\# (Fig. 3(b)), oriented triangular needles with base diameter of $\sim 5 \mu \mathrm{m}$ and length of $\sim 40 \mu \mathrm{m}$ were observed. At point 2\# (Fig. 3(c)), vertical hexagonal micro-rods with trunk diameter of $\sim 2.6 \mu \mathrm{m}$ and length of $\sim 8 \mu \mathrm{m}$ were observed. Thus, by decreasing the III/V ratio across the substrate, the nanostructures can change from triangular needles (1\#) to hexagonal micro-rods (2\#). This trend of morphology transformation is similar with that observed in sample A. As shown in the S3-S4 of the ESI, $\dagger$ the growth directions of needle and micro-rod are [1010] and [0001], respectively, which are confirmed by XRD measurement (S6 of the ESI $\dagger$ ).

\section{Sample B2}

Fig. 4(a) shows the optical photograph of sample B2, which was also grown with a substrate distance of $18 \mathrm{~mm}$. While the growth pressure was increased from 100 Torr to 200 Torr, which would further enhance the mixture of $\mathrm{TMGa}$ and $\mathrm{NH}_{3}$. As shown in Fig. 4(a), the surface color is uniform inside the growth region.

Fig. 4 shows the SEM images of sample B2, and vertical hexagonal micro-rods with an average trunk diameter of $\sim 3 \mu \mathrm{m}$ were observed across the growth region. With moving from the center (point $1 \#$ ) to the edge (point $2 \#$ ) of the growth region, the length of micro-rods decreases from $\sim 40 \mu \mathrm{m}$ (Fig. 4(b)) to $\sim 15$ $\mu \mathrm{m}$ (Fig. $4(\mathrm{c})$ ). In contrast with sample A and B1, there is no obvious morphology variation across the growth region, due to the enhanced mixture of the precursors via increasing the substrate distance and growth pressure.

\section{Discussion}

As shown in Fig. 5, NiAu droplet tends to leave the tip of the rod (Fig. 5(a)), otherwise the droplet would initiate a new growth of a branch (Fig. 5(b)), a polycrystalline structure (Fig. 5(c)) or a new rod (Fig. 5(d)) at the tip. According to previous report, ${ }^{7}$ the flat (0001) top surface will gradually shrink with the expanding of $\{1102\}$ sidewall facets. When the top surface becomes too small to support the NiAu droplet, the droplet would slide to the

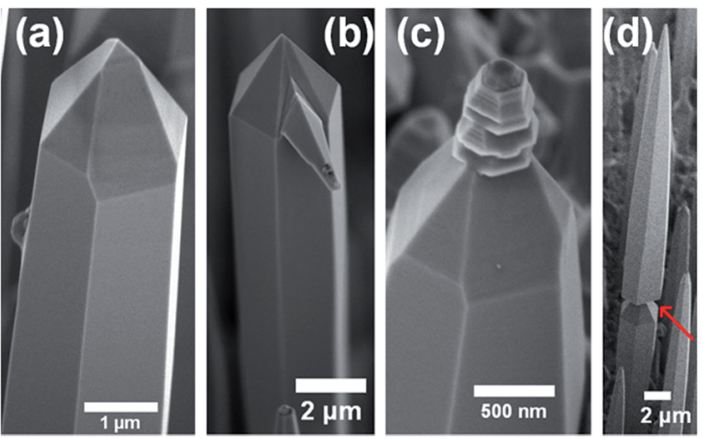

Fig. 5 The SEM images of the pillars (a) without NiAu droplet, or with a NiAu droplet at the tip for an additional growth of (b) a branch, (c) a polycrystal, and (d) a new pillar.

sidewall. Thus, except the initial stage of nucleation, the NiAu droplet should be not indispensable during the following growth. Moreover, for the nanowires (Fig. 2(d)) and needles (Fig. 3(b)), there is no droplet observed. It can be concluded that the $\mathrm{Ni} / \mathrm{Au}$ droplet would function as providing nucleation site rather than catalyst. ${ }^{15,24-26,29}$

As shown in Fig. 4, the GaN hexagonal micro-rods have a similar morphology with previously reported GaN rods, ${ }^{7-9,16}$ which were grown via SAG. Thus, the growth of micro-rods would have similar mechanism with SAG. In this case, NiAu would promote the pyrolysis of $\mathrm{NH}_{3}$ at a relatively low growth temperature $\left(790{ }^{\circ} \mathrm{C}\right)$, which is helpful for direct growth of nanodevices on unusual substrates of low melting temperature. ${ }^{30-35}$ While, the growth of nanowires (Fig. 2(d)) would have similar mechanism with VLS, because the nanowires have a high aspect ratio of $\sim 100$, which can not be realized by using normal SAG. ${ }^{7-9,16-18,20,22,23}$

The underlying growth mechanism in this case would be neither pure VLS ${ }^{1,2,12,13,24-27,29}$ nor pure SAG, ${ }^{7-9,16-18,22,23}$ because of the factors: (1) for VLS, metal droplet can normally be observed at the tip of nanowires, but in our case there is no droplet at the tip of nanowires (Fig. 2(d)) or needles (Fig. 3(b)); (2) for SAG,

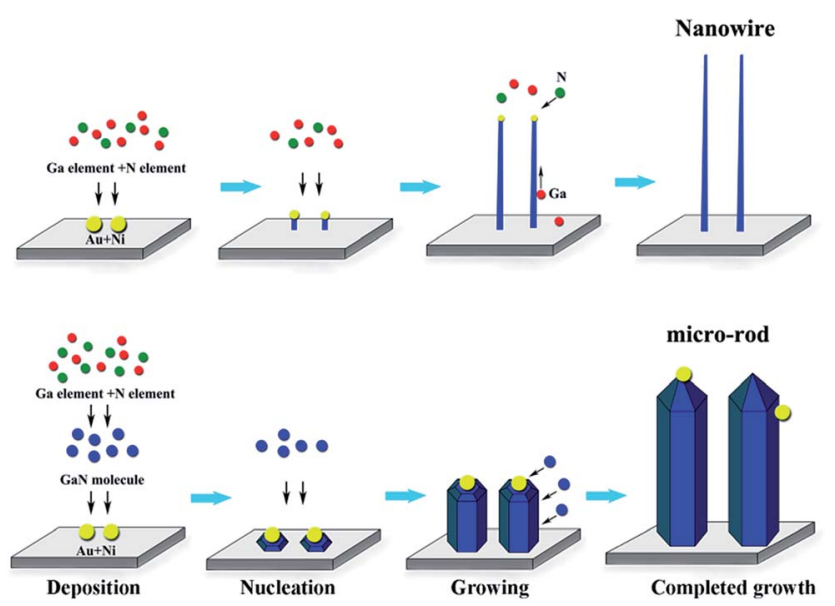

Fig. 6 The growth mechanism schematic of nanowires and microrods. 
metal catalyst is needless, but in our case the nanostructures can not grow without NiAu (not shown).

The growth mechanism schematic of the nanostructures was shown in Fig. 6. It is clear that the morphology variation from nanowire to micro-rod (Fig. 2) was mainly resulted from the decreasing of III/V ratio across the substrate. The III/V ratio was relatively high at center region, Ga element dominates and results in nanowire growth. When the III/V ratio is gradually decreased and the transport distance of Ga species is increased, the Ga species tends to react with $\mathrm{NH}_{3}$ and form GaN molecules in gas ambient due to the hot-wall reactor. Thus, there is a different growth mechanism, which includes: (1) the Ni/Au would play the role of providing nucleation site rather than catalyst; (2) the hot-wall MOCVD reactor enhances the prereaction between TMGa and $\mathrm{NH}_{3}$ in gas ambient, which produces the species of GaN molecule besides the Ga element; (3) the ratio between Ga element and GaN molecule is decided by the transport distance of Ga species; (4) Ga element dominates at center region and results in nanowire growth, while GaN molecule dominates at edge region and results in microrod growth. In-depth analysis will be carried out in our future study.

Fig. 7(a) shown the diameter distribution of sample A, B1 and B2. For sample A, the diameter is not continuously decreasing with III/V ratio decreasing from the center to the edge of the substrate. While for sample B1 and B2, the diameter was continuously decreasing with III/V ratio decreasing. The different variation trend of nanostructure diameter could be attributed to the difference of growth mechanisms. It can be seen from Fig. 2 that the nanostructure of sample A grown from position $1 \#$ to position $3 \#$ was nanowire which has similar mechanism with VLS, while from position $4 \#$ to $5 \#$, pyramid and micro-rod were observed, which have similar mechanism with SAG. So for the nanostructures grown under the same mechanism, the diameter decreased with decreasing III/V ratio from the center to the edge of the substrate. Fig. 7(b) shown the height distribution of three samples. For sample A, with decreasing III/V ratio, the height is decreasing except for the

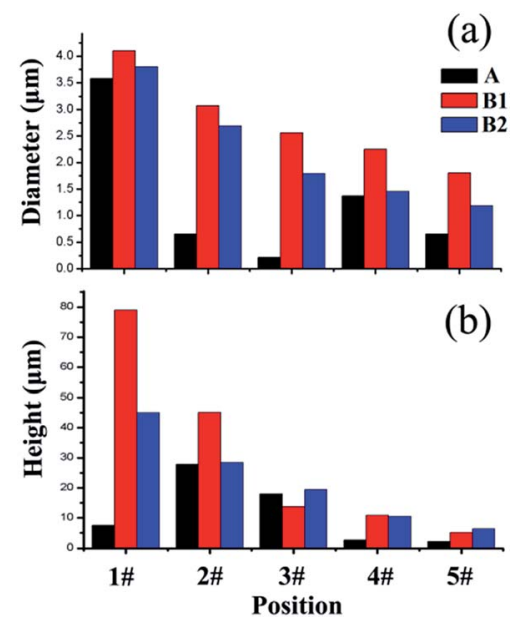

Fig. 7 The (a) diameter and (b) height distributions of sample A, B1 and B2 at different positions of the substrate. position 1\# where distorted large rods are observed. The short large rods are induced by very high III/V ratio and the lacking of N element for growth. For sample B1 and sample B2 (needles and micro-rods), the height has the same trend of change with localized III/V ration. In a word, both the height and diameter can be controlled by changing III/V ratio.

\section{Conclusions}

Different kinds of aligned GaN nanostructures (i.e., nanowires, needles and micro-rods) can be simultaneously grown on a single substrate, by naturally changing the III/V ratio across the substrate. The morphology variation from nanowires to micro-rods would be resulted from that the Ga species arriving at the substrate changes from Ga element to GaN molecule. Growth of these nanostructures on a single substrate at a temperature of $790^{\circ} \mathrm{C}$, which is much lower than that of SAG, is helpful for on chip integration of different kinds of nanodevices on unusual substrates of low melting temperature.

\section{Conflicts of interest}

There are no conflicts to declare.

\section{Acknowledgements}

This research was supported by grants from the New Century Excellent Talents in the University of China (NCET-05-0111), the International S\&T Cooperation Program of China (No. 2015DFR10970), and the National Natural Science Foundation of China (No. 61774027 and 61376050).

\section{References}

1 F. Qian, Y. Li, S. Gradečak, D. Wang, C. J. Barrelet and C. M. Lieber, Nano Lett., 2004, 4(10), 1975-1979.

2 T. R. Kuykendall, M. V. Altoe, D. F. Ogletree and S. Aloni, Nano Lett., 2014, 14, 6767-6773.

3 M. M. Rozhavskaya, W. V. Lundin, E. Y. Lundina, V. Y. Davydov, S. I. Troshkov, A. A. Vasilyev, P. N. Brunkov, A. V. Baklanov, A. F. Tsatsulnikov and V. G. Dubrovskii, J. Appl. Phys., 2015, 117, 024301.

4 R. Chen, T. Tran, K. Ng, W. Ko, L. Chuang, F. Sedgwick and C. Hasnain, Nat. Photonics, 2011, 5, 170-175.

5 L. Chuang, F. Sedgwick, R. Chen, W. Ko, M. Moewe, K. Ng, T. Tran and C. Hasnain, Nano Lett., 2011, 11, 385-390.

6 L. Chuang, M. Moewe, K. Ng, T. Tran, S. Crankshaw, R. Chen, W. Ko and C. Hasnain, Appl. Phys. Lett., 2011, 98, 123101.

7 A. Lundskog, U. Forsberg, P. O. Holtz and E. Janzén, Cryst. Growth Des., 2012, 12, 5491-5496.

8 Y. T. Lin, T. W. Yeh, Y. Nakajima and P. D. Dapkus, $A d v$. Funct. Mater., 2014, 24, 3162-3171.

9 K. Choi, M. Arita and Y. Arakawa, J. Cryst. Growth, 2012, 357, 58-61.

10 M. Munsch, N. S. Malik, E. Dupuy, A. Delga, J. Bleuse, J. M. Gérard, J. Claudon, N. Gregersen and J. Mørk, Phys. Rev. Lett., 2013, 110, 177402. 
11 P. Stepanov, A. Delga, N. Gregersen, E. Peinke, M. Munsch, J. Teissier, J. Mørk, M. Richard, J. Bleuse, J. M. Gérard and J. Claudon, Appl. Phys. Lett., 2015, 107, 141106.

$12 \mathrm{X}$. Zhou, J. Chesin, S. Crawford and S. Gradečak, Nanotechnology, 2012, 23, 285603.

13 Q. Li and G. Wang, Appl. Phys. Lett., 2008, 93, 043119.

14 G. Mitta and I. Lahiri, J. Phys. D: Appl. Phys., 2014, 47, 323001.

15 T. R. Kuykendall, A. M. Schwartzberg and S. Aloni, Adv. Mater., 2015, 27(38), 5805-5812.

16 B. O. Jung, S. Y. Bae, Y. Kato, M. Imura, D. S. Lee, Y. Honda and H. Amano, CrystEngComm, 2014, 16, 2273-2282.

17 P. M. Coulon, B. Alloing, V. Brändli, P. Vennéguès, M. Leroux and J. Zúñiga-Pérez, Appl. Phys. Express, 2016, 9, 015502.

18 J. Hartmann, X. Wang, H. Schuhmann, W. Dziony, L. Caccamo, J. Ledig, M. S. Mohajerani, T. Schimpke, M. Bähr, G. Lilienkamp, W. Daum, M. Seibt, M. Straßburg, H. H. Wehmann and A. Waag, Phys. Status Solidi A, 2015, 212, 2830-2836.

19 C. Tessarek, S. Figge, A. Gust, M. Heilmann, C. Dieker, E. Spiecker and S. Christiansen, J. Phys. D: Appl. Phys., 2014, 47, 394008.

20 R. Koester, J. S. Hwang, C. Durand, D. L. S. Dang and J. Eymery, Nanotechnology, 2010, 21, 015602.

21 X. J. Chen, B. Gayral, D. Sam-Giao, C. Bougerol, C. Durand and J. Eymery, Appl. Phys. Lett., 2011, 99, 251910.

22 C. G. Tu, C. Y. Su, C. H. Liao, C. Hsieh, Y. F. Yao, H. T. Chen, C. H. Lin, C. M. Weng, Y. W. Kiang and C. C. Yang, Nanotechnology, 2016, 27, 025303.

23 B. O. Juna, S. Y. Bae, S. Y. Kim, S. Lee, J. Y. Lee, D. S. Lee, Y. Kato, Y. Honda and H. Amano, Nano Energy, 2015, 11, 294-303.

24 B. Alloing and J. Zúñiga-Pérez, Mater. Sci. Semicond. Process., 2016, 55, 51-58.

25 V. G. Dubrovskii, N. V. Sibirev, J. C. Harmand and F. Glas, Phys. Rev. B: Condens. Matter Mater. Phys., 2008, 78, 235301.

26 J. Johansson, C. P. T. Svensson, T. Mårtensson, L. Samuelson and W. Seifert, J. Phys. Chem. B, 2005, 109, 13567-13571.
27 T. Kuykendall, P. Pauzauskie, S. Lee, Y. Zhang, J. Goldberger and P. Yang, Nano Lett., 2003, 3(8), 1063-1066.

28 H. Li, A. H. Chin and M. K. Sunkara, Adv. Mater., 2006, 18, 216-220.

29 W. C. Hou, T. H. Wu, W. C. Tang and F. C. Hong, Nanoscale Res. Lett., 2012, 7, 373.

30 Y. Sato, A. Fujiwara, S. Ishizaki, S. Nakane and Y. Murakami, Phys. Status Solidi C, 2017, 14, 1600151.

31 S. Y. Bae, J. W. Min, H. Y. Hwang, K. Lekhal, H. J. Lee, Y. D. Jho, D. S. Lee, Y. T. Lee, N. Ikarashi, Y. Honda and H. Amano, Sci. Rep., 2017, 7, 45345.

32 T. Itoh, A. Kobayashi, K. Ueno, J. Ohta and H. Fujioka, Sci. Rep., 2016, 6, 29500.

33 J. H. Choi, A. Zoulkarneev, S. I. Kim, C. W. Baik, M. H. Yang, S. S. Park, H. Suh, U. J. Kim, H. B. Son, J. S. Lee, M. Kim, J. M. Kim and K. Kim, Nat. Photonics, 2011, 5, 763-769.

34 J. H. Choi, J. Kim, H. Yoo, J. Liu, S. Kim, C. W. Baik, C. Cho, J. G. Kang, M. Kim, P. V. Braun, S. Hwang and T. S. Jung, Adv. Opt. Mater., 2016, 4, 505-521.

35 K. Chen, R. Kapadia, A. Harker, S. Desai, J. S. Kang, S. Chuang, M. Tosun, C. M. Sutter-Fella, M. Tsang, Y. Zeng, D. Kiriya, J. Hazra, S. R. Madhvapathy, M. Hettick, Y. Z. Chen, J. Mastandrea, M. Amani, S. Cabrini, Y. L. Chueh, J. W. Ager III, D. C. Chrzan and A. Javey, Nat. Commun., 2016, 7, 10502.

36 Z. Tian, Y. Li, X. Su, L. Feng, S. Wang, M. Zhang, W. Ding, Q. Li, Y. Zhang, M. Guo, F. Yun and S. W. Ricky Lee, Appl. Phys. Express, 2017, 10, 092101.

37 S. Y. Bae, K. Lekhal, H. J. Lee, T. Mitsunari, J. W. Min, D. S. Lee, M. Kushimoto, Y. Honda and H. Amano, J. Cryst. Growth, 2017, 468, 110.

38 J. Chen, P. Huang, X. Han, Z. Pan, C. Zhong, J. Liang, Z. Wu, Y. Liu and B. Zhang, Chin. Phys. B, 2017, 26, 068101.

39 M. Nami, R. F. Eller, S. Okur, A. K. Rishinaramangalam, S. Liu, I. Brener and D. F. Feezell, Nanotechnology, 2017, 28, 025202 . 